\title{
Impact of the pulse modulation format on distributed BOTDA sensors based on Simplex coding
}

\author{
Marcelo A. Soto, Gabriele Bolognini*, Fabrizio Di Pasquale \\ Scuola Superiore Sant’Anna, via G. Moruzzi 1, 56124 Pisa, Italy. *E-mail: g.bolognini@sssup.it
}

\begin{abstract}
We experimentally analyse the impact of pulse modulation format on BOTDA sensors exploiting Simplex coding. A careful optimisation of modulation format is required to avoid spurious oscillations causing severe penalties in the measurement accuracy.
\end{abstract}

Keywords: Stimulated Brillouin scattering, optical fibre sensors, temperature sensing, strain sensing, Simplex coding.

\section{INTRODUCTION}

Optical fiber sensors based on Brillouin optical time-domain analysis (BOTDA) allow for a highly accurate distributed detection of strain or temperature along a single-mode optical fiber, opening the way to a wide range of applications, ranging from civil and structural engineering to environmental monitoring and gas\&oil pipelines leakage detection. In last years, this topic has been the object of intense research activity worldwide, resulting in complex schemes including BOTDA-based configurations allowing for sub-meter resolution [1,2]. In addition, the use of optical pulse coding techniques [3] in BOTDA systems has been recently proposed [4], providing substantial benefits in terms of signal-tonoise ratio (SNR), without penalizing the sensor spatial resolution. BOTDA systems based on such schemes allow for enhanced sensing distances, reaching up to 50-km distance with $1-\mathrm{m}$ spatial resolution [4]. However, the use of long coded-pulse sequences to significantly improve the SNR, leads to new physical effects coming into play; in particular, the acoustic-wave dynamic plays a key role when a meter-scale spatial resolution is required, potentially leading to trace distortions and severe penalties in the measurement accuracy. Actually, the acoustic wave might be pre-excited to a different extent within the different codewords, affecting the Brillouin gain and leading then to an amplification of the probe signal which may significantly depend on the particular coded sequence. It is therefore necessary to carefully analyse this effect and its impact on the coded BOTDA system performance.

In this paper, we study such effect when using 10-ns pump pulses, evaluating the impact of the pulse modulation format in coded-BOTDA sensors. Experimental results clearly show that, without a careful optimisation of the modulation format, substantially different Brillouin gain levels can be experienced by the probe signal, depending on the different codeword traces, leading to distortions in the decoding process and to spurious oscillations affecting the sensors accuracy. Such detrimental effects can be effectively avoided by using an optimized non-return-to-zero (NRZ) modulation format, in which sufficiently small duty-cycle values effectively suppress the acoustic wave pre-excitation to a negligible level. Although our specific analysis is carried out considering Simplex coding, its validity is general and can be directly applied to other coding schemes, such as linear and correlation-based codes, where the issue of acousticwave pre-excitation is also expected to be critical.

\section{SIMPLEX-CODED BOTDA SENSORS}

BOTDA sensors are based on the stimulated Brillouin scattering (SBS) interaction taking place between two counterpropagating optical beams and an acoustic wave. In the conventional BOTDA gain configuration [4], a strong pulsed pump wave at a frequency $v_{0}$ interacts with a weak continuous-wave $(\mathrm{CW})$ beam that propagates in the backward direction, at a lower frequency $v_{0}-\Delta v$, the so-called probe signal. Energy transfer from the pump to the probe signal takes place along the fibre whenever pump and probe spatially overlap and the optical frequency difference, $\Delta v$, is within the local Brillouin gain spectrum (BGS). The maximum Brillouin gain actually occurs at a frequency difference called Brillouin frequency shift (BFS), which depends linearly on both temperature $(\Delta T)$ and strain $(\Delta \varepsilon)$ variations, according to

$$
\Delta \mathrm{BFS}=C_{v_{\mathrm{B}} \varepsilon} \cdot \Delta \varepsilon+C_{v_{\mathrm{B}} T} \cdot \Delta T
$$

Fourth European Workshop on Optical Fibre Sensors, edited by José Luís Santos, Brian Culshaw, José Miguel López-Higuera, William N. MacPherson, Proc. of SPIE Vol. 7653, 765332

(C) 2010 SPIE · CCC code: 0277-786X/10/\$18 - doi: 10.1117/12.866338 
where $C_{v B \varepsilon}=0.048 \mathrm{MHz} / \mu \varepsilon$ and $C_{v B T}=1.07 \mathrm{MHz} /{ }^{\circ} \mathrm{C}$ are the strain and temperature coefficients for BFS in silica fibres. In BOTDA sensors, the spatial resolution is proportional to the pump pulse duration and is ultimately limited to $\sim 1 \mathrm{~m}$ due to the acoustic phonon lifetime of $\sim 10 \mathrm{~ns}$ [1,2]; in order to achieve high spatial resolution, short pump pulses are then required. However, when using a pulse duration comparable to the acoustic phonon lifetime, the SBS interaction is drastically reduced, significantly decreasing the SNR of the measurement and leading to a maximum sensing range which is limited by the achieved dynamic range of the measurement. One possibility to improve the SNR of the measurement would be to increase the peak pulse power launched into the sensing fiber; however, the maximum peak power is limited by the onset of pump depletion and modulation instability. Thus, the use of optical pulse coding [3] has been recently proposed to increase the SNR of BOTDA sensors [4], allowing for an extended sensing range, not affecting the spatial resolution. Such a method is based on the linear dependence of the intensity variations of the received probe signal $\left(\Delta I_{C W}\right)$ with respect to the pump intensity, according to [4]:

$$
\Delta I_{C W}(t, \Delta v) \propto \int_{v_{g} t / 2}^{v_{g} t / 2+\Delta z} g_{B}(\xi, \Delta v) \operatorname{Ip}(\xi, \Delta v) d \xi
$$

where $v_{g}$ is the light group velocity, $\Delta z$ is the pump-probe interaction length (corresponding to one half of the pulse length), $g_{B}(\xi, \Delta v)$ and $I_{P}(\xi, \Delta v)$ are the Brillouin gain and the pump intensity, respectively, at a given position $\xi$.

While in the conventional single-pulse case a BOTDA trace is obtained with a single measurement only, in case of coded pulses the single-pulse BOTDA trace is reconstructed by decoding several coded traces, obtained sending different codewords. However, the Brillouin gain of a given pulse within each codeword may be affected by a pre-existing acoustic wave generated by previous pulses within the same codeword, leading to a possible detrimental trace distortion when using a pulse width of the order of the phonon lifetime, as for instance 10-ns pulses for 1-m spatial resolution. In that case, the intensity variation of the probe signal actually depends on the specific bit sequence (i.e. codewords), leading to possible distortion during trace decoding.

In the following sections we experimentally investigate the detrimental effect induced by the nonlinear SBS interaction described above, distorting the measured BGS when conventional 10-ns non-return-to-zero (NRZ) pulses are used in Simplex-coded BOTDA sensors. Then, the use of return-to-zero (RZ) Simplex-coded pulses is proposed and analysed for different duty cycles in order to provide a uniform SBS gain (irrespective of the specific codeword), suppressing the above-mentioned effect and allowing for a linear decoding process and undistorted BGS interpolation.

\section{EXPERIMENTAL SETUP}

The experimental setup reported in Fig. 1 has been used to analyse the impact of the pulse modulation format on Simplex-coded BOTDA sensors. The CWlight from a distributed-feedback (DFB) laser at 1550 $\mathrm{nm}$ with $\sim 10 \mathrm{dBm}$ output power, is split to generate both pump and probe beams. In the pump branch, an Erbium-doped fibre amplifier (EDFA) has been placed before a Mach-Zehnder modulator (MZM), in order to provide undistorted sequences of coded pulses with high extinction ratio. A computer-

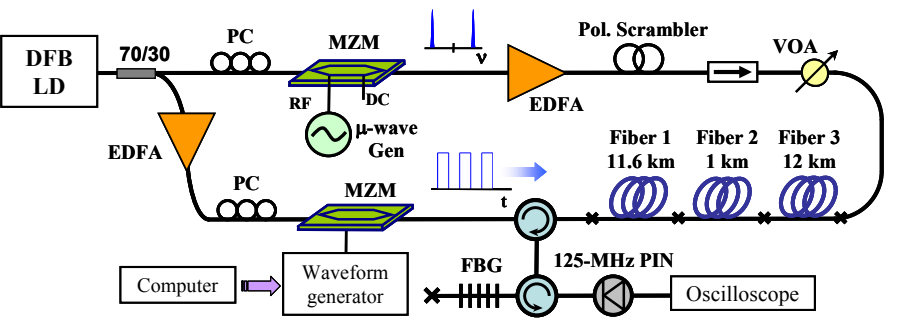

Fig. 1. Experimental setup for the Simplex-coded BOTDA-based sensor. controlled waveform generator is actually connected to the MZM to generate 127-bit Simplex codewords, with individual pulse duration of $10 \mathrm{~ns}$, providing an attainable spatial resolution of $1 \mathrm{~m}$. On the other branch, the probe signal is generated by intensity modulation of the $\mathrm{CW}$ light through a MZM controlled by a microwave (RF) generator and a DC bias voltage supplier. In order to measure the distributed BGS along the fibre, both sidebands, generated around the laser carrier frequency, are swept around the BFS by tuning the frequency of the microwave signal. Note that high carrier suppression can be obtained by a proper bias adjustment. In order to control the probe power level that is launched into the fibre, an EDFA and a variable optical attenuator (VOA) have been used. A polarization scrambler has also been employed to depolarize the probe signal and hence to reduce fluctuations of the Brillouin gain due to polarization changes along the fibre. The sensing fibre is composed of three single-mode fibre spools for an overall distance of $\sim 25$ $\mathrm{km}$. Although both sidebands of the probe are sent into the fibre, only the Stokes component is selected at the receiver side by using two optical circulators and a narrowband fibre Bragg grating (FBG, $<0.1 \mathrm{~nm}$ bandwidth). A 125-MHz PIN photodiode is connected to an oscilloscope, which is controlled by a computer to perform the measurements. 


\section{RESULTS AND DISCUSSIONS}

\subsection{Simplex-coded BOTDA sensor based on NRZ pulses}

In our study, we first analyse the impact of 127-bit Simplex-coded pulses with NRZ format on BOTDA sensors with 1-m spatial resolution. Fig. 2 reports six coded traces corresponding to codewords \#1, \#8, \#32, \#64, \#96 and \#108 of the standard S-Matrix [3]. It is evident from Fig. 2 that coded traces originating from different codewords are characterized by different amplification values and different intensity levels. Considering that the number of pulses (bits ' 1 ') is the same for every codeword, nonlinear amplification clearly occurs in such case. This can be explained by the fact that codewords containing long bursts of consecutive pulses (e.g. codeword \#64) actually give enough time to the acoustic wave to reach its maximum value, providing a large Brillouin amplification. On the other hand, codewords containing a uniform distribution of pulses, such as codeword \#1 (101010...101), are weakly amplified due to the continuous damping of the acoustic wave. Depending on the bit distribution within codewords, a notably different SBS amplification is obtained, as clearly shown in Fig. 2.

In order to analyse the impact of this nonlinear amplification mechanism on the decoded BGS, the temperature of the second and third fibre spools (see Fig. 1) has been changed to induce a shift in the BFS of $\sim 15 \mathrm{MHz}$ and $\sim 25 \mathrm{MHz}$ with respect to the first spool, which is kept at room temperature. Fig. 3 reports the decoded BGS vs

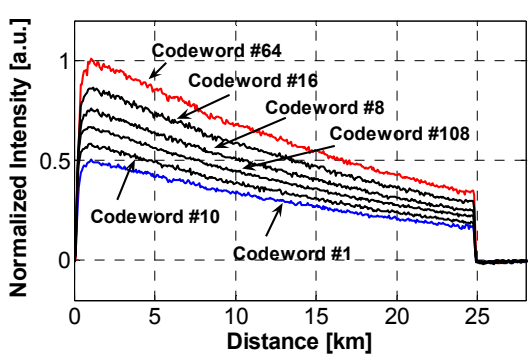

Fig. 2. Simplex-coded traces with NRZ pulses. distance, exhibiting evident distortion due to the presence of both undershoots and overshoots right after the temperature transitions. The distortion is originated in the linear decoding process by the nonlinear gain that affects the different coded traces. Such a distortion is also evident by looking at the BGS interpolated at different distances as reported in Fig. 4; we can clearly see a significant impact on the fitting process used to determine the BFS parameter. While the BGS shown in Fig. 4a at $11 \mathrm{~km}$, which is away from the temperature transition, actually exhibits an undistorted Lorentzian profile with a linewidth of $\sim 35 \mathrm{MHz}$, the high-gain traces in the decoding process lead to significant overlapping of the spatial information within the codewords, hence distorting the BGS near the temperature transitions. Actually trace oscillations occur along a distance corresponding to the code length (i.e. $127 \mathrm{~m}$ in this case) around BFS transition zones. We can clearly see in Figs. 4b and 4c that the decoded BGS actually exhibits a spurious oscillation near the frequency range corresponding to the BGS of the neighbouring fibre, leading to significant and unpredictable errors in the fitting.
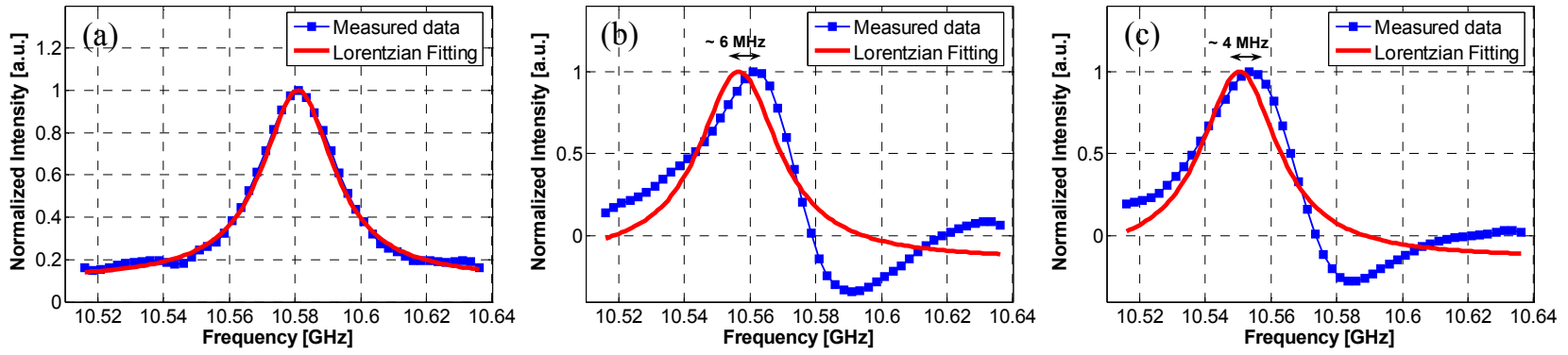

Fig. 4. BGS obtained with 127-bit Simplex coded NRZ pulses at a distance of (a) $11 \mathrm{~km}$ (Fibre 1), (b) 11.6 km, (Fibre 2) and (c) 12.6 km (Fibre 3).

\subsection{Simplex-coded BOTDA sensor based on RZ pulses}

In order to avoid the distortions previously observed when using Simplex coding with 10-ns NRZ pulses, we propose the use of RZ coded pulses for BOTDA sensors. Actually the impact of RZ pulses is analysed for different duty cycle values, looking at the maximum amplification difference between the traces relative to codeword \#1 and \#64 (as also shown in Fig. 2 for NRZ format). Fig. 5 actually reports the intensity difference between these two coded traces as a function of the pulse period and duty cycle; we can clearly observe that the trace difference decreases while reducing the duty cycle. In particular this difference becomes negligible when the separation between consecutives pulses is greater than $30 \mathrm{~ns}$ (i.e. greater than 3 times the acoustic decay time), corresponding to a duty cycle of less than $25 \%$ (40-ns pulse period). Fig. 6 actually shows the same coded traces reported in Fig. 2, but using RZ pulses with 16.7\% duty cycle. It is evident that a RZ modulation format characterized by a low duty cycle value allows for a uniform SBS amplification for all 
coded traces, providing $\sim 2 \%$ intensity difference on traces with different codewords. This behaviour demonstrates that the interaction of pump pulses with a pre-existing acoustic wave can be effectively suppressed when using RZ pulses with low duty cycle, leading to coded-trace intensities which are independent on the bit distribution.

As a consequence, when the BGS is measured using RZ Simplex-coded pulses

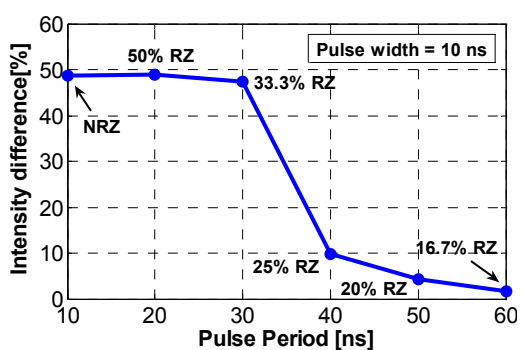

Fig. 5. Maximum relative intensity variation of Simplex-coded traces with NRZ and RZ pulses as a function of the pulse period.

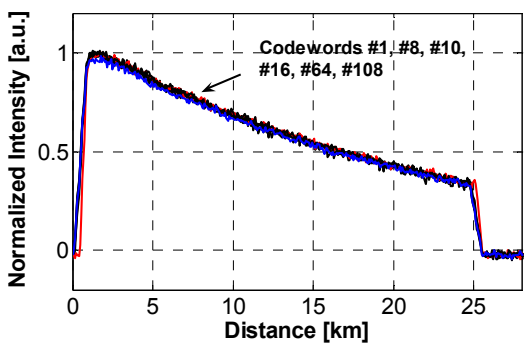

Fig. 6. Simplex-coded traces with RZ pulses and $16.7 \%$ duty cycle (equivalent to coded traces reported in Fig. 2 with NRZ pulses) with an optimised duty cycle, the decoded BGS along the fibre does not exhibit any distortion, as shown in Fig 7. This feature can also be observed in Fig. 8, where the measured and interpolated BGS at different distances along the fiber (corresponding to those of Fig. 4) are reported. Note that the effective BGS is given by the convolution of the natural Brillouin gain (exhibiting a Lorentzian profile with $\sim 20-35 \mathrm{MHz}$ linewidth [2]) and the pump pulse spectrum; hence, using 10-ns pulses, the BGS modifies to a Gaussian-like profile, which is well-described by a pseudo-Voigt profile obtained as a linear combination of both Lorentzian and Gaussian curves [5].

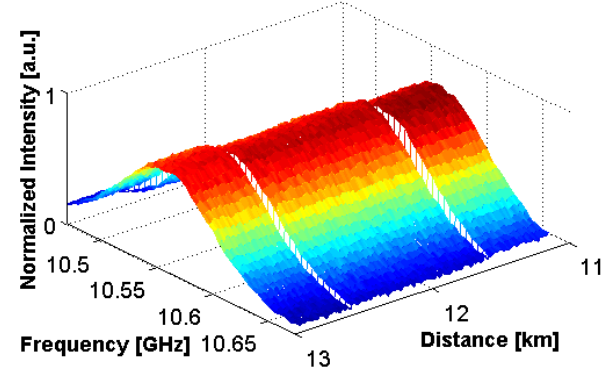

Fig. 8 clearly points out that the decoded BGS measured near the transition regions at the second and third fibre input (which correspond to $11.6 \mathrm{~km}$ and $12.6 \mathrm{~km}$, as in Figs. $4 \mathrm{~b}$ and $4 \mathrm{c}$ ) can be properly fitted by a pseudo-Voigt curve with a linewidth of $\sim 100 \mathrm{MHz}$, as expected when using standard BOTDA with 10-ns pulses [5].

It is important to note that, when using short pulses, the BGS broadening reduces the BFS measurement resolution, affecting the
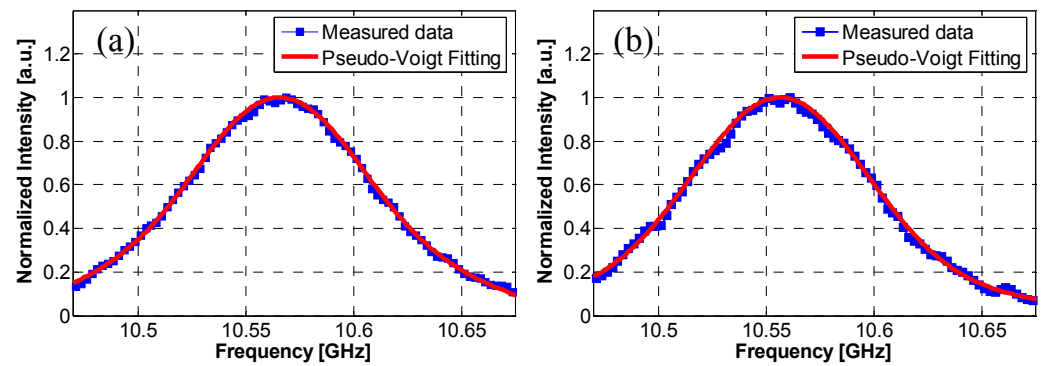

Fig. 8. BGS obtained with 127-bit Simplex coded RZ pulses at a distance of (a) $\sim 11.6 \mathrm{~km}$, in Fibre 2, and (b) $\sim 12.6 \mathrm{~km}$, in Fibre 3. performance of both coded and standard BOTDA sensors with meter-scale spatial resolution [2]. However, in our proposed system the measurement SNR is enhanced thanks to the use of RZ Simplex-coded pulses, providing $\sim 7.5 \mathrm{~dB}$ SNR improvement in case of 127-bit code length, and leading then to an enhanced performance without distortion.

\section{CONCLUSIONS}

In conclusion we analysed the impact of the pump modulation format on coded-BOTDA sensors. A detrimental effect, involving acoustic-wave pre-excitation within coded traces, and leading to severe distortion of the decoded BGS near BFS transition regions, has been observed and analysed. A solution, consisting in the use of low duty-cycle RZ pulses, has been proposed, achieving an effective suppression of code-induced distortions. Careful optimisation of the pulse modulation format allows for a full exploitation of coding gain, offering $7.5 \mathrm{~dB}$ SNR enhancement over 25 -km distance.

\section{REFERENCES}

1. L. Thévenaz et al., "Distributed fiber sensing using Brillouin echoes," Proc. of SPIE vol. 7004, paper 70043N, 2008.

2. Y. Dong et al., "12-km distributed fiber sensor based on differential pulse-width pair BOTDA," Proc. of SPIE vol. 7503, paper 75032G, 2009.

3. M. D. Jones, "Using Simplex codes to improve OTDR Sensitivity," IEEE Photon. Technol. Lett. 15(7), 822-824, 1993.

4. M. A. Soto et al., "Simplex-coded BOTDA fiber sensor with $1 \mathrm{~m}$ spatial resolution over a $50 \mathrm{~km}$ range," Opt. Lett. 35(2), 259-261, 2010.

5. X. Bao et al., "Characterization of the Brillouin-loss spectrum of single-mode fibers by use of very short $(<10$-ns $)$ pulses," Opt. Lett. 24(8), 510-512, 1999. 